Rev. Latinoam. Psicopat. Fund., São Paulo, v. 11, n. 3, p. 365-379, setembro 2008

\title{
Considerações históricas sobre a difusão do pensamento kleiniano no Brasil*
}

Jorge Luis Ferreira Abrão

Este artigo tem por objetivo apresentar os diferentes momentos que caracterizaram a difusão do pensamento kleiniano nas Sociedades de Psicanálise do Brasil. Trata-se de uma pesquisa histórica de natureza qualitativa, realizada com base em entrevistas com 13 psicanalistas que participaram dessa difusão. As primeiras influências surgiram a partir de 1950, por intermédio de pioneiros que fizeram formação na Inglaterra ou na Argentina. As áreas em que os kleinianos foram pioneiros - psicanálise de crianças e análise de psicóticos - constituíram-se nos primeiros focos de utilização deste referencial no país. De 1950 a 1970, evidenciou-se uma apreensão dogmática das idéias kleinianas. A partir de 1980, com o advento de novas traduções da obra de Melanie Klein, e com a introdução do pensamento kleiniano contemporâneo, constata-se uma utilização mais equilibrada deste modelo teórico-técnico.

Palavras-chave: Psicanálise, história, Brasil, Melanie Klein

* Artigo elaborado a partir da tese de doutorado intitulada A tradição Kleiniana no Brasil: uma investigação histórica sobre a difusão do pensamento kleiniano, defendida em 2004 no Instituto de Psicologia da USP. 
Segundo Phyllis Grosskurth (1992), Melanie Klein mostrou-se preocupada, no final de sua vida, com a sobrevivência de sua obra. No entanto, com o passar dos anos, o sistema conceitual kleiniano mostrou-se bastante sólido e revigorado, seja por intermédio das ampliações teóricas introduzidas pelos seguidores mais próximos ou pela expansão do interesse pelas idéias kleinianas, em diferentes partes do mundo, tanto na América Latina, onde elas tiveram grande aceitação, quanto em regiões que outrora foram refratárias a esta vertente do pensamento psicanalítico, como os Estados Unidos e a França.

No Brasil, ao longo do período histórico que marca a difusão da psicanálise, encontraremos uma crescente participação do pensamento kleiniano, que tem sido tomado como um importante modelo de referência e fonte de inspiração para orientar a prática clínica e fundamentar as reflexões teóricas de um número expressivo de analistas que compõem as diversas Sociedades Psicanalíticas filiadas à IPA no Brasil. ${ }^{1}$ Esta afirmação enseja algumas contextualizações que serão objeto deste artigo: particularmente é necessário que se explicite a forma e os meios pelos quais o pensamento kleiniano foi introduzido no Brasil, a maneira com que estas idéias foram assimiladas e empregadas neste meio e, ainda, os desdobramentos sofridos por este modelo teórico e técnico no país.

Com o intuito de atingir os objetivos propostos, realizamos uma pesquisa de caráter histórico concernente à difusão do pensamento kleiniano nas Sociedades de Psicanálise existentes no Brasil, tomando como referência os fundamentos metodológicos da "Historia Nova" (Le Goff e Nora, 1988a, 1988b, 1988c). Para tal, realizamos 13 entrevistas com profissionais represen-

1. Empregaremos a expressão "Sociedades de Psicanálise" no plural para designar o conjunto das instituições psicanalíticas filiadas à IPA (International Psychoanalytic Association) existentes no Brasil. 
tativos de diferentes momentos históricos da psicanálise brasileira, e que se destacaram no ensino das idéias kleinianas ou na produção teórica e clínica a partir deste referencial.

Salvo ocorrências isoladas, fruto da idiossincrasia de alguns intelectuais como o médico e antropólogo Arthur Ramos, que em 1933 publicou um artigo intitulado "A technica da psychanalyse infantil", no qual apresentava os fundamentos da análise infantil, fazendo referências a Melanie Klein e a seus trabalhos, as idéias kleinianas surgiram no Brasil com maior contundência a partir de meados da década de 1950 .

Os primeiros contatos dos psicanalistas brasileiros com o referencial kleiniano se fizeram por duas vias: inicialmente através de alguns profissionais que realizaram sua formação psicanalítica no exterior e, posteriormente, pela presença de analistas estrangeiros no país.

Neste sentido, durante a década de 1950 observamos um forte movimento imigratório entre os profissionais dedicados à psicanálise no Brasil, que se dirigiam aos principais centros produtores de conhecimento psicanalítico na época, particularmente a Inglaterra, e em alguns casos particulares a Argentina, a fim de se aperfeiçoarem. Assim o fizeram Décio Souza e Manoel Lyra, da Sociedade Brasileira de Psicanálise do Rio de Janeiro, Virgínia Bicudo, Lygia Amaral e Frank Philips, da Sociedade Brasileira de Psicanálise de São Paulo, e Mário Martins e Zaira Martins, da Sociedade Psicanalítica de Porto Alegre.

$\mathrm{O}$ reconhecimento deste fluxo imigratório e da aproximação com as idéias kleinianas dele decorrentes é amplamente reconhecido, mesmo fora do contexto brasileiro. A afirmação de Hinshelwood (1992) ao relatar as especificidades do Grupo Kleiniano corrobora este raciocínio. Para esse autor, “... da década de $50 \mathrm{em}$ diante houve um considerável interesse em 'uma formação kleiniana' e muitas pessoas chegaram de outros países para fazer formação psicanalítica, especialmente da América do Sul...” (p. 350).

Posteriormente, particularmente a partir de meados da década de 1960, evidenciamos um maior interesse dos analistas estrangeiros pela psicanálise brasileira, o que resultou na presença constante, no Brasil, de psicanalistas de diversas partes do mundo, convidados a exercer atividades propedêuticas. Assim, da Sociedade Britânica de Psicanálise temos Hanna Segal, Herbert Rosenfeld, Wilfred Bion, Paula Heimann, Donald Meltzer, Betty Joseph e Elizabeth Spillius, e da Associação Psicanalítica Argentina encontramos Arminda Aberastury, Eduardo Kalina, Mauricio Knobel e Raquel Soifer.

Partindo destes primeiros contatos travados com o referencial kleiniano, podemos antever algumas conclusões que caracterizam a introdução e o desenvolvimento das idéias de Melanie Klein no Brasil. 
Ao considerarmos a forma como as idéias kleinianas foram introduzidas nas instituições psicanalíticas, observamos algumas linhas de regularidade que permitem definir ao menos duas características comuns a este momento histórico: os primeiros psicanalistas brasileiros que empregaram e divulgaram o pensamento kleiniano, definidos como pioneiros, notorizando-se também como fundadores de suas Sociedades e o caminho percorrido por esses profissionais foi bastante semelhante: inicialmente travaram contato com a teoria kleiniana no exterior e, posteriormente, introduziram esse conhecimento em suas Sociedades, ao exercerem funções didáticas nessas instituições.

Outra conclusão a ser destacada refere-se à origem das informações relativas à teoria kleiniana, que começaram a circular no Brasil a partir da década de 1950. Fundamentalmente, a introdução dessas idéias foi proveniente de duas matrizes institucionais distintas: a Sociedade Britânica de Psicanálise e a Associação Psicanalítica Argentina.

O ciclo imigratório para a Inglaterra, em busca de formação psicanalítica, tem se constituído em uma invariante da psicanálise brasileira, haja vista que desde o início da formação das Sociedades Psicanalíticas até a atualidade é possível identificar diferentes gerações de psicanalistas que cruzaram o Oceano Atlântico tendo como destino a Sociedade Britânica de Psicanálise. Evidentemente as contingências que aproximaram esses profissionais do Grupo Kleiniano, em um lapso de tempo de aproximadamente cinqüenta anos, são variadas e merecem ser diferenciadas.

Assim, temos elementos para supor que os primeiros profissionais que rumaram para a Inglaterra o fizeram fundamentalmente pelo prestígio granjeado pela Sociedade Britânica de Psicanálise naquele período, visto que essa instituição destacava-se como o principal pólo gerador e dispersor de conhecimento psicanalítico no mundo pós-guerra. Ao narrar a história das Sociedades de Psicanálise no período da Segunda Guerra Mundial, com base nos arquivos da IPA, Adam Limentani (1990) conclui, a respeito da Inglaterra:

Se é verdade que viver numa Inglaterra devastada pela guerra começava a perturbar a vida da sociedade, não houve seguramente nenhum efeito negativo visível sobre a criatividade científica de seus membros. Foi naqueles anos que Melanie Klein escreveu seus estudos pioneiros sobre o luto e sua relação com os estados maníaco-depressivos (1940), e sobre o complexo de Édipo à luz das primeiras angústias (1945). Nesse mesmo período é dada à luz a introdução de "Uma psicopatologia revisada das psicoses e das neuroses" por Fairbairn, e assim sucessivamente. Houve também estudos importantes assinados por P. Heimann, E. Glover, J. Bowlby, S. Isaacs etc. (p. 37) 
Uma vez em contato com a Sociedade Britânica de Psicanálise, a aproximação dos profissionais brasileiros com o Grupo Kleiniano se deu gradualmente, a partir de interesses pessoais que foram despertados durante a estada na Inglaterra e pela forte e marcante presença que Melanie Klein e seus partidários exerciam junto à Sociedade.

Nas gerações mais recentes de profissionais que se encaminharam para a Inglaterra durante as décadas de 1970 e 1980, evidenciamos uma situação diferenciada, uma vez que nesses casos prevaleceu um interesse preliminar pela teoria kleiniana suscitado pelo contato com esta vertente teórica no país, o que estimulou o desejo de uma aproximação com o Grupo Kleiniano desde o início. A chegada ao Brasil desta segunda geração de analistas formados a partir do referencial kleiniano demarca uma nova etapa da difusão do pensamento kleiniano, dadas as inovações e as iniciativas por eles empreendidas, tanto no campo didático quanto editorial, conferindo maior vitalidade a esse referencial teórico.

Um movimento em sentido contrário, mediante a presença de analistas do Grupo Kleiniano no Brasil, oscilou de uma existência efêmera, fruto de acontecimentos fortuitos ocorridos ao longo dos anos de 1950 e 1960, para ocorrências regulares, durante as décadas de 1970 a 1990. Um bom exemplo pode ser evidenciado no caso de Bion, que teve uma presença marcante no país, sobretudo na Sociedade Brasileira de Psicanálise de São Paulo, tendo visitado a cidade em 1973, a do Rio de Janeiro e de São Paulo em 1974, a de Brasília no ano de 1975 e novamente São Paulo em 1978.

Com relação à Associação Psicanalítica Argentina, o período de maior intercâmbio com a psicanálise brasileira está compreendido entre meados da década de 1940 e meados da década de 1970. Evidenciamos nos primeiros anos um movimento centrífugo em que profissionais do Rio de Janeiro e do Rio Grande do Sul dirigiram-se à Argentina com a finalidade de fazer formação psicanalítica e, posteriormente, um movimento centrípeto, em que vários analistas argentinos foram convidados a ministrar cursos, palestras e supervisões no Brasil.

Particularmente entre 1945 e 1955, Buenos Aires converteu-se em uma rota de imigração percorrida com freqüência por profissionais do Rio de Janeiro e de Porto Alegre que desejavam fazer formação psicanalítica, haja vista que a institucionalização da psicanálise nessas regiões ocorreu de forma mais lenta e conturbada do que a evidenciada em São Paulo. A opção pela Associação Psicanalítica Argentina, que a esta altura já contava com algum prestígio e notoriedade, conquistados, entre outros fatores, pela presença de vários analistas europeus que haviam se radicado na Argentina, como Angel Garma, Marie Langer, Willy Baranger, deveu-se ao menos a dois fatores. O primeiro refere-se à proximidade geográfica com o Brasil, o que estimulou, sobretudo os profissionais 
do Rio Grande do Sul, a rumarem para Buenos Aires. O segundo fator diz respeito ao distanciamento mantido pela Associação Psicanalítica Argentina com relação às disputas institucionais e políticas existentes entre os diferentes grupos formadores de psicanalistas que, na época, iniciavam suas atividades no país. Isto foi particularmente significativo para um grupo de profissionais do Rio de Janeiro que, por ausência de afinidades com o Grupo de Estudos já estruturado naquela cidade, preferiu imigrar para a Argentina com o intuito de se qualificarem como analistas.

A participação do referencial kleiniano no ciclo de formação oferecido pela Associação Psicanalítica Argentina sofreu muitas variações ao longo dos anos: no início, entre as décadas de 1940 e 1950, prevalecia uma orientação rigorosamente freudiana; pouco mais tarde, entre as décadas de 1960 e 1970, os trabalhos de Melanie Klein começam a ganhar mais espaço, sobretudo pela iniciativa de jovens analistas que buscavam nessa autora respostas para as suas indagações clínicas, entre os quais podemos destacar Marie Langer, León Grinberg, Willy Baranger, José Bleger, David Liberman, entre outros. Um setor dentro da Associação Psicanalítica Argentina em que a teoria kleiniana foi adotada, desde o início, como tendência hegemônica é facilmente identificado no grupo dedicado à psicanálise de crianças, capitaneado por Arminda Aberastury, que congregava em torno de si um séqüito de adeptos e seguidores. Esse grupo teve importante participação na estruturação da psicanálise de crianças a partir do referencial kleiniano em toda a América Latina, no Brasil inclusive. No período compreendido entre 1965 e 1975, a presença dos analistas argentinos ligados à psicanálise de crianças foi constante em São Paulo, no Rio de Janeiro e em Porto Alegre, realizando conferências, ministrando cursos e dando supervisões.

Uma vez reconhecidos os caminhos pelos quais as idéias kleinianas foram introduzidas no Brasil, cabe apresentar a forma como este pensamento foi apreendido e utilizado no país.

Como demonstramos, no momento em que as idéias kleinianas começaram a ser introduzidas, já existiam no país algumas Sociedades de Psicanálise estruturadas, o que pressupõe a existência de uma prática clínica razoavelmente sedimentada. Neste sentido, o novo referencial teórico e técnico foi primeiramente empregado em áreas da psicanálise ainda pouco exploradas, ou abordadas com limitações e precariedade, como a psicanálise de crianças e a análise de pacientes psicóticos, áreas nas quais Melanie Klein e seus seguidores foram pioneiros.

Com relação à psicanálise de crianças, a presença das idéias kleinianas foi uma unanimidade em todas as Sociedades existentes no país, sobretudo entre as décadas de 1950 e 1970. A presença de alguns pioneiros, que haviam mantido contato direto com Melanie Klein e seus seguidores na Inglaterra, e o intercâmbio constante com o grupo liderado por Arminda Aberastury, criou as condições 
necessárias para que a psicanálise de crianças se desenvolvesse em São Paulo, no Rio de Janeiro e em Porto Alegre, a partir do referencial kleiniano.

No que concerne ao tratamento de psicóticos, as evidências indicam que muitos analistas brasileiros recorreram isoladamente ao referencial kleiniano como substrato para o trabalho com este tipo de paciente; no entanto, do ponto de vista institucional, não encontramos uma unanimidade nacional como o observado no caso da psicanálise de crianças, tendo em vista que esta forma de apropriação foi estabelecida com maior nitidez na Sociedade Psicanalítica de Porto Alegre do que nas demais instituições psicanalíticas existentes no país. Essa situação pode ser explicada pelo fato de a Sociedade Psicanalítica de Porto Alegre ter mantido um intercâmbio direto com a Faculdade de Medicina de Porto Alegre, de tal forma que os cursos de especialização e residência médica em psiquiatria foram fortemente influenciados pela psicanálise, conservando um forte acento kleiniano. Por outro lado, o mesmo tipo de intercâmbio entre instituições psicanalíticas e faculdades de medicina não foi evidenciado com tanta nitidez em São Paulo e no Rio de Janeiro.

Embora tenha sido conferido particular destaque a duas áreas específicas, é importante destacar que a abrangência desse modelo de pensamento psicanalítico não ficou circunscrita unicamente a esses domínios; ao contrário, ganhou paulatinamente espaço na maioria das Sociedades de Psicanálise, influenciando de forma geral a prática da psicanálise.

Ao ser incorporado na prática clínica brasileira, o pensamento kleiniano foi sendo, ao longo dos anos, assimilado, interpretado e transformado no contexto nacional, de forma que a apreensão deste sistema conceitual passou por algumas variações que oscilaram entre uma compreensão enrijecida e estereotipada até a constituição de características próprias.

Inicialmente, em um período que pode ser delimitado - unicamente para efeitos didáticos - entre as décadas de 1950 e 1970, prevaleceu uma compreensão e uma utilização das idéias kleinianas marcadas por um viés estereotipado e dogmático. Em síntese, isto significa que algumas características nodais do modelo teórico e técnico proposto por Melanie Klein ganharam contornos superlativos, sendo entendidas de maneira descontextualizada e empregadas de forma demasiadamente rígida.

Evidências desta conclusão podem ser encontradas tanto na conduta técnica empregada na época, que valorizava interpretações em termos de objetos parciais, enfatizava de forma demasiada os aspectos agressivos da personalidade, em detrimento dos componentes libidinais, e propunha a manutenção de um setting excessivamente rígido; quanto ao entendimento teórico que, segundo Elias Rocha Barros (1995), se pautava por uma apreensão de conceitos desvinculada do 
contexto histórico e institucional em que foram gerados, de tal sorte que idéias de uma fase inicial do trabalho de Melanie Klein foram tomadas, em determinados momentos, como a versão final de seu pensamento.

A conjugação de vários fatores contribuiu para que a situação descrita acima se materializasse em determinado momento da difusão das idéias kleinianas no Brasil.

A origem das primeiras informações sobre a obra de Melanie Klein decorre de um período político bastante polarizado dentro da Sociedade Britânica de Psicanálise, em que alguns exageros foram cometidos com o intuito de demarcar as diferenças entre kleinianos e freudianos. Essa característica refletiu-se na produção teórica de alguns analistas iniciantes, fato este constatado por Elizabeth Spillius (1983) que analisou todos os Kleinian Menbership Papers da década de 1950 e início de 1960, a fim de identificar as características da técnica kleiniana empregada na ocasião. Suas conclusões podem ser resumidas da seguinte forma:

There are the occasional strikingly original exceptions, but most of the papers of the 1950 s and the early 1960 s seemed to me rather doctrinaire and inclined to emphasize the patient's destructiveness in a way that might have felt persecuting to the patient. A second feature is that unconscious phantasies were evidently interpreted to the patient very directly and in part-obejct language. In the earliest papers there were very few references to countertransference and to projective identification, especially to projection of aspects of the patient and of his internal objects into the analyst. (p. 324-325)

Ao equacionarmos todos os fatores apontados - que conferem uma forma bastante peculiar ao Grupo Kleiniano na Inglaterra ao longo da década de 1950 - somos levados a concluir que a criatividade, a inovação e a originalidade de pensamento que nortearam as atividades de figuras destacadas desse grupo psicanalítico fazendo-o conviver de forma muito próxima com um pensamento clínico estagnado e marcado por clichês que, na ânsia de afirmar-se como kleiniano, muitas vezes desfigurava a verdadeira natureza das idéias apresentadas por Melanie Klein.

Possivelmente, com o passar dos anos e através do contato direto com Melanie Klein e seus discípulos mais próximos, essas distorções e exasperações, frutos de uma compreensão de primeira hora matizada por contingências políticas e administrativas, foram cedendo espaço para uma apreensão mais equilibrada e condizente com os fundamentos do pensamento kleiniano.

Assim, tanto os brasileiros quanto os argentinos que nessa ocasião mantiveram contato regular com o Grupo Kleiniano, receberam uma instrução sobre o tema marcada por deformações, que o distanciamento institucional e o limitado contato com a tradição oral não permitiram corrigir a contento. Uma vez no 
Brasil, esses psicanalistas reproduziram as exasperações e as exacerbações que caracterizaram a formação obtida no exterior, devido ao contexto histórico em que se inseriram.

Por outro lado, é possível constatar que as formas de transmissão do pensamento kleiniano no Brasil, tanto por vias orais quanto escritas, contribuíram para uma compreensão aleijada deste sistema conceitual.

A transmissão oral, por meio de cursos, palestras e supervisões, enquanto recursos recorrentes do ensino psicanalítico, foi marcada por uma ênfase exagerada em determinados aspectos da teoria e da técnica kleiniana, com o intuito de demarcar fronteiras e conferir aos adeptos desse sistema conceitual maior identidade e legitimidade em sua prática clínica.

Com relação às vias de transmissão escrita, devemos dedicar particular atenção às traduções das obras de Melanie Klein para o português, que começaram a surgir a partir de 1969, nas quais são encontrados vários problemas: via de regra, elas foram malcuidadas, não se preocupando com a homogeneização de determinados conceitos entre as várias publicações de editoras diferentes, e a seqüência das publicações não seguiu uma ordem cronológica, dificultando uma compreensão longitudinal da obra de Melanie Klein. Isso se explica pelo fato de essas traduções terem sido feitas a partir das primeiras versões das obras da autora, e não com base nas obras completas publicadas na Inglaterra em 1975, em que cada trabalho é acompanhado por uma nota explicativa e a seqüência cronológica de sua publicação é respeitada. No artigo intitulado "The problem of originality and imitation in psychoanalytic thought: a case study of kleinian thinking in Latin America", publicado em 1995, Elias Rocha Barros faz uma apreciação altamente depurada da produção kleiniana na América Latina, além de dedicar algumas linhas para comentar a qualidade e as condições em que os textos de Melanie Klein foram traduzidos para o português e o espanhol. De acordo com as conclusões desse psicanalista,

The first translations of Klein's works into Portuguese and Spanish were in general of low quality (with the exception of a few published in Argentina) and were based on her complete works published in 1948, which is in itself an incomplete edition, and not on the 1975 edition authorised by The Melanie Klein Trust. In de Spanish-speaking countries, the chronological order in which Melanie Klein's works were originally published was at least respected. In Brazil, on the other hand, no particular chronological order was followed in translating and publishing her works. The resulting scattered order in which the translations were made along with translations based on early, unrevised edition introduced considerable conceptual confusion. (p. 840)

Rev. Latinoam. Psicopat. Fund., São Paulo, v. 11, n. 3, p. 365-379, setembro 2008 
Ao aliarmos as constatações relativas à precariedade das traduções dos textos de Melanie Klein no país às dificuldades inerentes ao próprio pensamento kleiniano, como, por exemplo, a falta de uma sistematização da obra que permita acompanhar e compreender suas transformações conceituais ocorridas ao longo do tempo, podemos asseverar que a aproximação dos leitores brasileiros com os textos de Melanie Klein resultou em dificuldades de compreensão conceitual, sobretudo nos casos de contatos superficiais, nos quais o açodamento era a característica reinante.

A inversão desta perspectiva, com o surgimento de uma compreensão menos distorcida da teoria kleiniana, e de uma prática clínica menos carregada de estereótipos, mais independente e inovadora, começa a ser evidenciada a partir da década de 1980 . Vários fatores contribuíram para a emergência desta nova situação, entre os quais destacamos os mais relevantes.

A ocorrência de um novo ciclo de intercâmbio com a Sociedade Britânica de Psicanálise - seja por um maior interesse dos analistas britânicos em realizarem visitas regulares ao Brasil com a finalidade de apresentar uma visão contemporânea das idéias kleinianas, como o fizeram, por exemplo, Hanna Segal, Herbert Rosenfeld, Wilfred Bion, Betty Joseph e Elizabeth Spillius, ou pela ocorrência de um novo ciclo de imigração para a Inglaterra, desencadeado por profissionais mais jovens em busca de formação psicanalítica, ou mesmo de aperfeiçoamento profissional junto ao Grupo Kleiniano, como é o caso de Elias Mallet da Rocha Barros, Elizabeth Lima da Rocha Barros, Liana Pinto Chaves, Maria Elena Salles de Brito, Otávio Salles e Luiz Meyer - favoreceu a transmissão de uma visão atual e mais equilibrada dos trabalhos de Melanie Klein e seus seguidores, garantiu o incremento do ensino e proporcionou o aprimoramento da prática clínica.

Outro fator a destacar diz respeito aos esforços editoriais capitaneados por Elias Rocha Barros a partir da década de 1990. Ao promover a publicação das Obras Completas de Melanie Klein, com base na edição inglesa de 1975, e tornar disponível, em português, vários trabalhos de autores kleinianos contemporâneos, ele possibilitou ao leitor brasileiro uma visão mais abrangente e completa do conjunto de idéias que fundamentam a teoria e a técnica kleiniana na atualidade e criou condições para uma visão histórica de suas transformações.

Em estudo retrospectivo, realizado na Sociedade Psicanalítica de Porto Alegre sob a coordenação de Cláudio Eizirik, em que foram analisadas as características da produção psicanalítica entre os anos de 1963 a 1995, por intermédio dos trabalhos de admissão para membro associado da referida Sociedade, identificouse a forte prevalência do referencial kleiniano; Melanie Klein é a autora mais citada depois de Freud ao longo das quatro décadas investigadas. No tocante à transformação do trabalho clínico nesse período, constatou-se que na última década, quando comparada com as primeiras, o analista, segundo Eizrik et al. (1999) “... 
considera mais a realidade externa, está menos centrado na agressão, interpreta menos a sexualidade, interpreta mais a auto-estima, pergunta mais e revela uma interpretação mais equilibrada da transferência positiva e negativa" (p. 217).

Partindo desta evolução concernente à apreensão e à utilização das idéias kleinianas no Brasil, somos reportados ao debate relativo ao destino tomado pelo pensamento kleiniano no contexto nacional, sobretudo com relação à originalidade, à criatividade e à singularidade destas idéias.

É um dado consensual que a transmissão da teoria psicanalítica sofreu inúmeras transformações ao longo dos anos, na medida em que foi difundida para diferentes partes do mundo. Diz Mezan (2002):

O que ocorre, a meu ver, é que a psicanálise vai se adaptando às circunstâncias sociais, econômicas e culturais tanto da sua clientela como daqueles que a praticam, e não poderia ser de outra forma, já que, estando no mundo, é afetada por aquilo que se passa neste mundo, e por sua vez o afeta segundo certas vias; e não é inútil tentar precisá-las. (p. 317)

Neste processo de difusão, encontraremos peculiaridades que marcam a inserção da psicanálise em diferentes países, em função de características locais. No Brasil, um dos problemas mais evidentes que tem mobilizado os debates relativos ao tema da psicanálise nacional está centrado no binômio reprodução/originalidade de idéias psicanalíticas.

Apenas para efeito didático, podemos estabelecer, com base no que foi apresentado até este ponto, três etapas, ou momentos, que balizaram o desenvolvimento de um pensamento kleiniano inovador no contexto da psicanálise brasileira, quais sejam: a prática intuitiva, o reconhecimento das transformações e a sistematização teórica.

No primeiro momento, observamos a ocorrência de pequenos ajustes ou modificações na prática clínica, feitos de forma intuitiva e quase imperceptível à primeira vista, com o intuito de tornar o processo analítico mais adaptado às necessidades e às demandas da dupla analítica, que vão sendo constituídas ao longo do trabalho. A característica nodal neste momento é que algumas modificações vão sendo introduzidas na clínica psicanalítica sem que se tenha obrigatoriamente um olhar reflexivo e crítico sobre essas ocorrências que podem, por vezes, sobretudo no caso de analistas menos experientes, ser negadas conscientemente. Dentro desta configuração, a referência para o trabalho psicanalítico é sempre o sistema teórico dominante, tomado como modelo de identificação; assim sendo, qualquer modificação introduzida é sempre entendida como desvio ou transgressão a ser evitada, o que dificulta o reconhecimento das modificações que pouco a pouco vão sendo introduzidas. Assim constatou o psicanalista Sérvulo Augusto Figueira (1991): 
... a importação psicanalítica cria um dilema interessante, pois as transformações que o saber importado sofre não são pensáveis a partir das definições e das autorepresentações também importadas. O resultado de tudo isso é que certas realidades da nossa psicanálise acabam na posição de duplamente negadas, sendo sua percepção, portanto, duplamente impedida. (p. 117)

Em um segundo momento, denominado de reconhecimento das transformações, acompanhamos o surgimento de um maior juízo e reflexão sobre as modificações, as adaptações e as ampliações que os analistas foram introduzindo individualmente em sua prática clínica, e uma conseqüente reflexão sobre esta nova conjuntura, entendida ora como inovação, ora como desvio e transgressão. Nesta etapa encontramos um reconhecimento mais explícito das inovações incorporadas ao trabalho clínico e, mais do que isto, a convicção das necessidades destas transformações ou, ao menos, de parte delas; no entanto, a fidelidade ao modelo teórico dominante torna o reconhecimento público desta condição indesejado ou temido pelas críticas e efeitos negativos que supostamente ocorreriam.

O terceiro momento, delimitado sob a alcunha de sistematização teórica, constitui-se em uma etapa de organização de dados, experiências e informações, e conseqüente divulgação, por intermédio da publicação de trabalhos científicos, das transformações identificadas na prática clínica, de forma a compartilhar experiências e discutir os efeitos - sejam eles positivos ou não - que estas modificações exercem sobre a psicanálise. Cabe, neste momento, propor elaborações teóricas que dêem conta de explicar conceitualmente as modificações que foram incorporadas no trabalho psicanalítico desenvolvido no país ou em dada região, apontando as conexões e as dissidências com relação ao modelo teórico dominante, de tal modo que se estimulem o debate científico e a delimitação de um referencial psicanalítico nacional.

Tomando como referência as etapas por nós apresentadas, é possível sustentarmos o seguinte raciocínio: a partir do sistema kleiniano, adotado por muitos psicanalistas como referência teórica e modelo de identificação, foram sendo promovidas modificações e adaptações na prática clínica com o objetivo de fazer frente às vicissitudes que o trabalho prático impunha aos profissionais, na medida em que o modelo teórico forjado na Europa foi transposto para o contexto nacional. No entanto, ao invés de se constituírem em inovações, que a princípio o são, estas modificações foram tomadas como desvios em relação à norma, devido à adesão canônica ao modelo original, entendido como parâmetro a ser seguido.

Embora não seja possível definir uma produção legitimamente brasileira, é plausível sustentarmos a conclusão de que a prática e a produção teórica desen- 
volvidas no país com base nas idéias kleinianas preservam traços de singularidade e originalidade que lhes conferem uma identidade própria ainda pouco mapeada. Em linhas gerais, e de forma bastante simplificada, podemos considerar que os principais nichos de criatividade e originalidade da psicanálise de inspiração kleiniana no Brasil estão situados no campo da técnica, que se expressam, em termos práticos, em duas categorias: uma maior permeabilidade às contingências ambientais durante o processo psicanalítico e a adoção de um setting mais flexível, sem que isto signifique uma banalização da técnica. No entanto, estas variações ou inovações da técnica psicanalítica, aqui sugeridas, merecem uma investigação mais apurada.

Realizamos neste artigo um breve esboço capaz de alinhavar os diferentes aspectos e momentos que caracterizaram a difusão do pensamento kleiniano no Brasil, ainda que o tema seja vasto e que os desdobramentos das questões suscitadas requeiram pesquisas mais aprofundadas.

\section{Referências}

EIzIRIK, C.L. et. al. Algumas modificações na prática clínica da SPPA: um estudo retrospectivo. Revista de Psicanálise da Sociedade Psicanalítica de Porto Alegre, Porto Alegre, v. 6, n. 2, p. 205-225, 1999.

FigueirA, S.A. A dimensão teórico-clínica da psicanálise no Brasil: imitação ou criação. Revista Brasileira de Psicanálise, São Paulo, v. 25, n. 1, p. 109-122, 1991.

Grosskurth, P. O mundo e a obra de Melanie Klein. Rio de Janeiro: Imago, 1992.

Hinshelwood, R. Dicionário do pensamento kleiniano. Porto Alegre: Artes Médicas, 1992.

Le Goff, J.; Nora, P. História: novos problemas. 3. ed. Rio de Janeiro: Francisco Alves, 1988a.

. História: novas abordagens (3. ed.). Rio de Janeiro: Francisco Alves, 1988b.

. História: novos objetos (3. ed.). Rio de Janeiro: Francisco Alves, 1988c.

LimentANI, A. O movimento psicanalítico no mundo durante os anos de guerra (19391945) segundo os arquivos da A. P. I. In: Revista Internacional de História da Psicanálise. Rio de Janeiro: Imago, 1990. p. 33-40.

Mezan, R. Psicanálise e cultura, psicanálise na cultura. In: Interfaces da psicanálise. São Paulo: Companhia das Letras, 2002. p. 317-392.

Ramos, A. A technica da psychanalyse infantil. Archivos Brasileiros de Hygiene Mental, Rio de Janeiro, v. 1, n. 2, p. 195-205, 1933. 
Rocha BARRos, E.M. The problem of originality and imitation in psychoanalytic thought: a case study of kleinian thinking in Latin America. International Journal of Psychoanalysis, Londres, v. 76, n. 4, p. 835-843, 1995.

SpILlius, E.B. Some developments from the work of Melanie Klein. International Journal of Psychoanalysis, Londres, v. 64, n. 3, p. 321-332, 1983.

\section{Resumos}

(Consideraciones históricas sobre la difusión del pensamiento kleiniano en el Brasil)

Este artículo tiene el objetivo de presentar los diferentes momentos que caracterizan la difusión del pensamiento kleiniano en las Sociedades de Psicoanálisis de Brasil. Se trata de una investigación histórica de naturaleza cualitativa, realizada y fundamentada en entrevistas con trece psicoanalistas que participaron de esa difusión. Las primeras influencias surgieron en el año de 1950, por intermedio de pioneros que se formaron en Inglaterra y Argentina. Las áreas en que los kleinianos fueron pioneros - el psicoanálisis de niños y de psicóticos - se constituyeron en los primeros focos de utilización de este referencial en el pais. Desde 1950 hasta 1970, se evidenció una aprehensión dogmática de las ideas kleinianas. A partir de 1980, con la llegada de nuevas traducciones de la obra de Melanie Klein y con la introducción del pensamiento kleiniano contemporáneo, se constata una utilización más equilibrada de este modelo teórico-técnico.

Palabras clave: Psicoanálisis, historia, Brasil, Melanie Klein

(Considérations historiques sur la diffusion de la pensée kleinienne au Brésil)

Cet article a pour but de présenter les différents moments qui ont caractérisé la diffusion de la pensée kleinienne dans les Sociétés de Psychanalyse du Brésil. Il s'agit d'une recherche historique de nature qualitative réalisée au moyen d'interviews avec treize psychanalystes qui ont participé à cette diffusion. Les premières influences se sont manifestées à partir de 1950, à travers les pionniers qui avaient étudié en Angleterre ou en Argentine. Les domaines dans lesquels les Kleiniens ont été pionniers - la psychanalyse des enfants et l'analyse des psychotiques - ont formé les premiers foyers d'application de cette théorie au Brésil. De 1950 à 1970, on observe une assimilation dogmatique des idées kleiniennes. À partir de 1980, grâce à l'avènement des nouvelles traductions de l'oeuvre de Melanie Klein et à l'introduction de la pensée kleinienne contemporaine, on constate une utilisation plus équilibrée de ce paradigme théoriquetechnique.

Mots clés: Psychanalyse, histoire, Brésil, Melanie Klein 
(Considerations on the diffusion of Kleinian thought in Brazil)

This article describes the different moments that characterized the diffusion of Kleinian thought in psychoanalytic societies in Brazil. The article results from qualitative historical research based on interviews with thirteen psychoanalysts who participated in this process of diffusion. The first influences appeared in 1950, exercised by pioneers trained either in Britain or in Argentina. The areas in which the Kleinians were pioneers - psychoanalysis of children and of psychotics - were the first aspects dealt with by this group in Brazil. Between 1950 and 1970 very dogmatic approaches were taken toward Kleinian ideas. As of 1980, with the publication of new translations of Melanie Klein's work and with the introduction of contemporary Kleinian thought, a more balanced use of this theoretical-technical model could been seen.

Key words: Psychoanalysis, history, Brazil, Melanie Klein

Versão inicial recebida em dezembro de 2006

Versão aprovada para publicação em julho de 2008

Jorge Luis Ferreira Abrão

Professor do Departamento de Psicologia Clínica e do Programa de Pós-Graduação em Psicologia e Sociedade da Faculdade de Ciências e Letras - FCL/Universidade Estadual Paulista - UNESP-Assis, SP, Brasil.

Av. Rui Barbosa, 1262/91

19814-000 Assis, SP, Brasil

e-mail: abro@assis.unesp.br

Rev. Latinoam. Psicopat. Fund., São Paulo, v. 11, n. 3, p. 365-379, setembro 2008 